\title{
The Look of the Link - Concepts for the User Interface of Extended Hyperlinks
}

\author{
Harald Weinreich, Hartmut Obendorf, Winfried Lamersdorf \\ University of Hamburg, Department of Informatics, VSYS \\ \{weinreich, 5obendor, lamersdorf\}@informatik.uni-hamburg.de
}

\begin{abstract}
The design of hypertext systems has been subject to intense research. Apparently, one topic was mostly neglected: how to visualize and interact with link markers.

This paper presents an overview of pragmatic historical approaches, and discusses problems evolving from sophisticated hypertext linking features. Blending the potential of an XLink-enhanced Web with old ideas and recent GUI techniques, a vision for browser link interfaces of the future is being developed. We hope to stimulate the development of a standard for hyperlink marker interfaces, which is easy-to-use, feasible for extended linking features, and more consistent than current approaches.
\end{abstract}

\section{Keywords}

XLink, distributed hypertext, user interfaces, link markers, Web

\section{INTRODUCTION}

As part of a recent endeavor to extend the functionality of the WWW, the World Wide Web Consortium (W3C) proposed XLink, XPointer and XPath [DeRose 2000, DeRose 2001, Clark 1999] as linking standards for the Extended Markup Language (XML). While these specifications elaborately define the syntax of XML linking, visualization of and interaction with extended links remain largely undefined. Although current user perception of hypertext is mainly determined by Web experience and as a result links naturally seem to be blue and underlined, previous hypertext systems demonstrated other link visualization and interaction techniques. Some of these techniques do not only show the text in a more legible way, they will also be better applicable to complex anchors.

Historical approaches can help to find solutions for the Web of the future. A first look at link visualizations will help to become familiar with the associated problems. XML linking will bring fundamental changes in the way links work; therefore we try to examine the use of historical approaches for XML linking and attempt to find out where they fall short. Furthermore, we present a set of proposals for link visualization, thus hoping to fuel the discussion about the user interface of one of today's most important applications.
HISTORICAL APPROACHES TO LINK VISUALIZATION

Though hypertext systems frequently offer advanced functionality, the essence of hypertext are machine-supported links that provide rapid access to modularized information nodes in non-sequential order [Conklin 1987, Shneiderman \& Kearsley 1989]. The visualization of link anchors by link markers and the interaction with links - the link user interface - are an important characteristic of usable hypertext systems. However, no standard link user interface exists and only few papers reflect on this topic.

When visualizing hyperlinks, two conflicting objectives must be balanced well: on the one hand the information presented has to be intelligible and the design should be as little restricted as possible, on the other hand links have to be marked and emphasized to be noticed and identified.

Links originating from graphics or parts of graphics are especially problematic. They can only be marked by changing the presentation of the original image. Historically, only few methods to mark graphics were explored, one reason being that in early systems the focus was usually put on text support. Embedded graphics did not have a dominant rank as link anchors as in the Web today. A system that supported links from images was Intermedia. It marked anchors by an arrow icon above the image [Yankelovich et al. 1988]. Mosaic's approach was dreadful: it drew thick blue boxes around all linked graphics. Serious conflicts with intended page design forced the majority of Web authors to hide the border. Instead, the hypermedia designer has to use other, non-standardized methods to make anchors visible. Often, 3D-elements, boxes, and rollovers are used to mark anchors. However, this can only work when the graphics are specifically created for the purpose of providing link anchors. Later changes in anchor placement would force to alter the graphics.

For textual anchors two objectives have to be balanced well: on the one hand the readability of the text has to be kept as good as possible, on the other hand the link anchors have to be distinguishable from other text. The appearance of text has been changed in a variety of ways to highlight the anchor region: typeface, style, size and color of the text have been altered, and graphical elements like arrows or boxes have been added.

Some systems, like Peter J. Brown's Guide [Brown 1987], used different text styles like bold and italic types to mark anchors. This overloads the typographic significance of these attributes and limits the formatting options for unmarked text. 


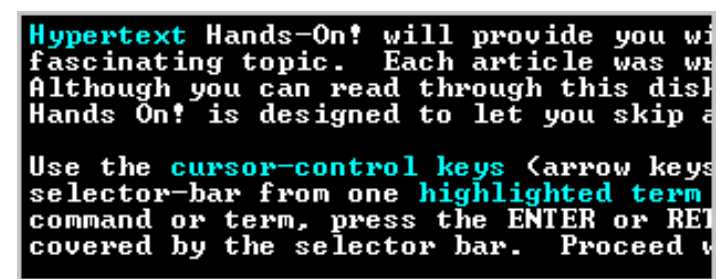

Figure 1: HyperTIES used cyan to highlight links [from Shneiderman \& Kearsley 1989]

HyperTIES (Fig. 1) avoided this problem by using a distinct text color for link markers, similar to Hyper-G's browser Harmony (Fig. 5) which utilized background colors [Shneiderman \& Kearsley 1989; Andrews 1996]. This has the advantage that the typeface and style of the text can be chosen freely.

Waterland
Graham Swift's 1983 novel Waterland,
according to Michael Wood of The New York
Review of Books, "is a formidably intelligent
book ...animated bv an imnressive. angrv nitv at
Figure 2: Intermedia's link marker arrows

IRIS' "Intermedia" marked hyperlinks with little arrow icons between lines of text (Fig. 2), showing the start of the link span but not its endpoint [Yankelovich et al. 1988]. "Emacs Info" brackets link markers with asterisk symbols. These methods occupy extra screen space and change the layout of the text by inserting additional elements.

\begin{tabular}{|c|}
\hline 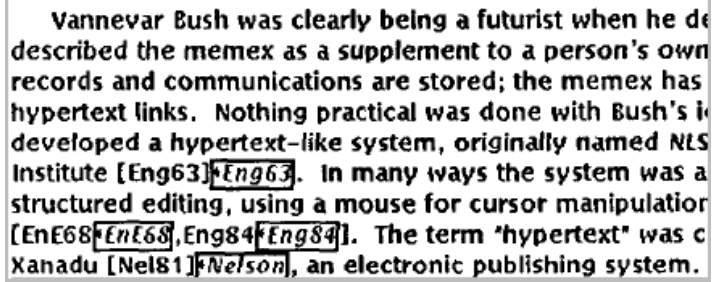 \\
\hline \\
\hline
\end{tabular}

Bernstein's Hypergate, the Neptune hypertext system (Fig. 3) and some early Web browsers UdiWWW (Fig. 4) drew boxes around the link marker text. This works with the layout but is quite obtrusive and distracting. An improvement to this technique could be found in HyperCard and Storyspace. They drew the boxes only when the reader pressed particular keys, making links evident on request thus keeping the text pristine the rest of the time.

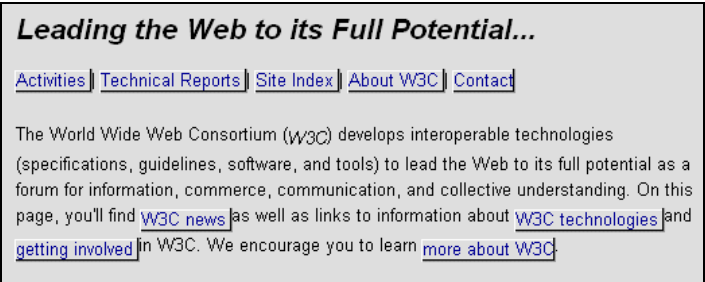

Figure 4: Anchor highlighting in UdiWWW
In fact, this was the consensus solution after the Hypertext '87 demo sessions, when hypertext designers could first compare all existing systems side by side [Bernstein 1996]. Hiding links has many advantages: pages stay uncluttered, text stays readable, and page design is less influenced by link appearance. However, when using "links on demand" the interface designer must be aware of a potential disadvantage: since links are not always visible, possibly distracting mode switches have to be applied. Therefore this link trigger has to be seamlessly integrated into the interface.

A less desirable variant of this method was used by Symbolics Document Examiner [Walker 1987]: link markers were hidden so well that they were only highlighted when the mouse passed over them. This forces a "hunt and peck" search for active regions. In Microcosm and DLS the user can query the system for invisible links by marking a word or some text and then issue a search for matching links [Carr et al. 1995]. Though anchors are not marked, this model is well applicable to generic links, given that most words can be selected as anchors.

Ignoring previous experiences, Mosaic returned to colored (blue) and underlined text to indicate link markers. This is not the optimal solution as it emphasizes the link marker text permanently. The underlined markers stick out from the surrounding text and decrease its readability as underlining interferes with descenders, letters that drop below the line like $\mathrm{p}, \mathrm{q}$ and $\mathrm{j}$. The blue color is also an imperfect choice, since especially elderly people have problems to perceive it; the human eye is less sensitive to the color blue than to other colors [Lythgoe, 1979]. The reasons for Mosaic's link marker appearance were obviously of technical nature: it was quite simple to implement, and at that time most computers had only 16 colors or a black and white display. Blue was the darkest of the available colors, closest to the black text; for monochrome displays, the text was underlined [NCSA 1996].

The pervasiveness of the Web has led us to accept underlined colored text as the de facto marker standard for all mainstream hypertext systems today. It can be found in help systems on various platforms and even in operating system components like the Microsoft Windows Explorer $(\mathrm{C}$. Though the use of a standard is desirable from a consistency point of view, user interfaces have otherwise improved, and we are still wedged with this historical hack.

More recent technologies like Cascading Style Sheets [Bos et al. 1998] allow to define the appearance of text links in various ways. Also, the look of links can be configured somehow in current browsers, however, the standard setting is still blue and underlined, and links on demand are still not possible. Even worse, for the visualization of link maps in graphics no standard method can be found nor is implemented in Web browsers. This shows how important it is to design user interfaces well, considering earlier experiences, and that even an interface with obvious design mistakes can become a standard. 


\section{ENTER XML LINKING}

While the concept of links made it possible to create nonsequential texts, rich hypertext systems offer a more sophisticated functionality, i.e., they support comprehensive structuring, editing and navigation features [Yankelovich et al. 1988; Bieber et al. 1997]. However, to date the Web itself only supports embedded one-way links. This limitation made the authoring of Web pages and the development of Web servers and browsers simple, enabling the Web to grow extremely fast. On the other hand, all approaches that try to integrate some extended functionality into the Web have to utilize workarounds to overcome the weaknesses of this simple approach. It was also hard to compete with the well-known standard browsers, as these often were better suited to display existing stylishly designed Web pages.

With the advent of XML linking, the Web will be able to offer many of the features hypertext experts are missing today [Vitali \& Bieber 1999]. Standard browsers already migrate to XML and XSLT [Clark 1999] and hopefully XML linking will become widely available soon.

The linking potential of XML linking is based on two key standards which are necessary to create and describe links and link anchors:

XLink itself defines links as relation between resources or portions thereof. Syntactically, a link consists of an arbitrary number of resources (local or remote) and arcs. A resource is any addressable unit of information or service, while arcs create directed connections between two resources each [DeRose, Maler, Orchard 2000].

XPointer allows to address different kinds of spans in XML documents [see DeRose, Maler, Daniel 2001]. They can vary from points to complex regions and can even be distributed over the document, e.g. a XPointer could be used to address the string "Goethe" in all citations in an XML file:

xpointer (string-range(//cite, "Goethe")).

Two types of semantic attributes are defined: machinereadable information is stored in the role and arcrole attributes and the corresponding human-readable textual information is kept in title attributes. This type information can be specified for the link as a whole, each endpoint of a link and for every arc.

XML linking also presents a solution for linking into other authors' read-only material, by addressing parts of the documents' structure. There is no need for tailored target anchors, which are embedded in the target document, any more. The importance of this can be seen from printed media, i.e., referring to distinct pages or paragraphs.

1 XML linking may not only be used for hypertext links, but for any kind of application describing relations, associations or compositions of XML documents. However, this paper focuses on its use for hypertext.
To summarize, XML linking will allow a multitude of new hyperlink features, among them:

- structure and contents may be separated;

- links may be bi-directional;

- links may be typed;

- links may have multiple endpoints;

- anchors may be complex or overlap.

While the syntax of XLink has been elaborately defined, most presentation and behavioral aspects of links have been deliberately excluded from the model. Only few hints on how to implement and interact with these features can be found in the XLink definition, and few ideas exist to enable the user to cope with this extended functionality. There is a vague notion of displaying title attributes to enrich link anchors semantically and computing role attributes to realize typed links [DeRose, Maler, Orchard 2000]. Pop-up windows are suggested for links with multiple endpoints [DeRose 1989; Bosak \& Bray 1999]. Though this might suggest that not much has changed, for the user interface, these new features create a lot of new questions for link visualization.

The chances seem to be good that XLink will succeed. The changes in browser capabilities since Mosaic suggest that more extensive hypertext features will eventually be accepted: Forms, JavaScript, Java Applets and Flash animations are now widely used. Some extended link functionality is already being simulated with DHTML, showing popup menus with multiple destinations or extra information.

\section{VISUALIZING EXTENDED HYPERTEXT FEATURES}

The two well-known hypertext models Dexter [Halasz 1990] and HyTime [Derose \& Durand 1994] had features almost matching, and sometimes exceeding XLink, but no system ever fully implemented them [Grønbæk \& Trigg 1999 , p. 42]. Nonetheless, many systems existed that were far ahead of their time and offered functionality that is not available in the Web today.

This enables us to find ideas and detect problems by looking at all these systems and how they implemented and visualized hyperlinks. We map the user interfaces of these programs to the linking features that are made available with the introduction of XLink. We can thus discuss what is needed by the user to profit from the extended functionality.

\section{Separation of Structure and Content}

Several former Open Hypermedia Systems like Microcosm/ DLS [Carr et al. 1995] or the Devise Hypermedia System [Grønbaek \& Trigg 1999] permitted to store links separately from documents in dedicated linkbases. Likewise, XLink will allow the separation of structure and content for the Web.

The external storage of links permits multiple linkbases to be used for single Web pages. These links may originate from the original author but also from other authors without 
write access to the original document, like a single user or members of a group ${ }^{2}$.!

The use of several different linkbases can result in an unintentionally great number of links $\mathrm{s}^{\frac{13}{4}}$. Therefore, the user must be enabled to select the employed linkbases. An example for such a method can be found in "Third V_ice", a browser plug-in that adds annotations to Web pages ${ }^{4}$ (Fig. 5). A part of its functionality is a service that adds links from an external linkbase to keywords. These annotation link markers are distinguished by orange underlines. Third Voice offers an extra tool bar in the head of the browser where the presentation of its links can easily be toggled.

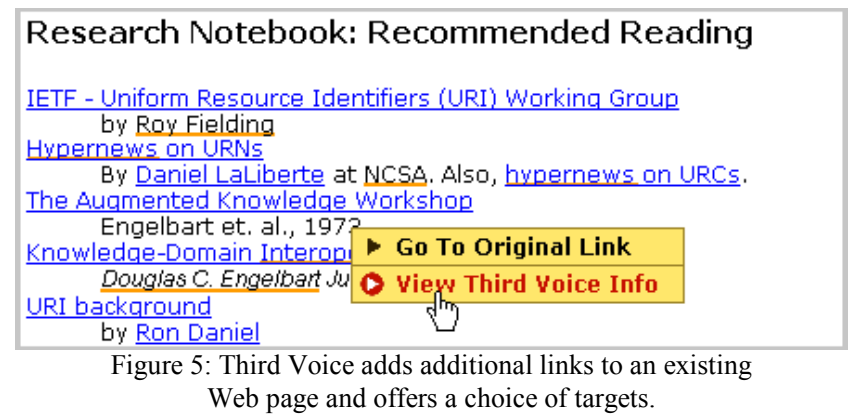

Microcosms/DLS already permitted the use of several linkbases. It offered a configuration screen to select the utilized link database from a given set [Carr et al. 1996]. Unfortunately this menu was not directly integrated into the browser interface and the addition of new linkbases was quite complicated. An XLink browser will also need the potential to find new linkbases ${ }^{5}$ and add them to a personal list. So far, no standard means has been established to do so.

\section{Bi-directional Links}

From the technical point of view bi-directional links help to keep links consistent and to avoid broken links. From the usability viewpoint they also permit to follow links backwards as opposed to the uni-directional "goto" links of the Web. A user could use this feature to find e.g. more recent information which is referring to an old but valuable document.

To benefit from bi-directional linking, the user interface has to support the backward traversal of links. Most hypertext systems with bi-directional links like Sepia, MacWeb or Hyper-G offered a "local map", showing nodes and connecting links. This visualizes the topology and permits the user to select source objects directly on the map.

2 These additional links can be used to annotate and supplement the existing information with other information of personal importance.

${ }^{3}$ These links may also overlap (see following sections).

${ }^{4}$ Third Voice is available at http://www.thirdvoice.com

${ }^{5}$ Furthermore, the primary linkbase will frequently change when browsing the Web, as it usually will be provided by the server hosting the current document.
For the Web, the retrieval of links that refer to the current document poses a serious problem. A prototype Web browser tool described in [Chakrabarti et al. 1999] gathered this information from search engines. They alternatively proposed to extend the HTTP protocol to send backlink information gathered from the referrer URLs in the server $\log$. The prototype offered a list of titles of Web documents that were linking to the current document.

Both approaches have their limitations if the number of links is high. Especially graphical maps use a lot of screen space if dozens of nodes and links are displayed. Thus, the number of objects has to be limited, e.g. by filtering the most appropriate ones.

\section{Typed Links}

A link type describes the relationship between source and destination of a link, often derived from semantic categories like "explanation" or "example" [Trigg 1983]. They were introduced to help users to get a better idea of a link target. Streitz et al. list semantic link information as their first principle of useful hypermedia system design [Streitz et al. 1992]. However, typed links are only helpful if the user can distinguish the different types.

Tim Berners-Lee's WWW proposal [Berners-Lee 1989] included typed links, and HTML allows Web authors to set the link type attributes rel and rev. Though, this feature is not supported by any current Web browser.

Sepia [Streitz et al. 1992] and MacWeb [Nanard \& Nanard 1993] displayed the link type in an overview map close to the arrow visualizing the link. Once more, this link information is only available to the user if he considers two areas at the same time: a document view and a link map. He has to join these two information segments cognitively.

Other systems use text style to distinguish different link anchor types: the current Microsoft help system displays explanatory pop-up links in green with a dotted underline and uses icons to indicate specific actions as the execution of a program. However, the potentials of Text style are quite limited, and inline icons can be distracting and create problems with the layout.

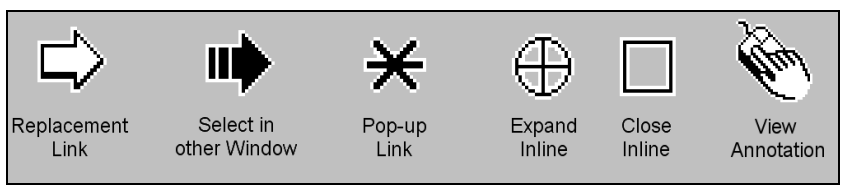

Figure 6: Different mouse pointers utilized by the Guide system.

The Guide system utilized different mouse pointers to make link characteristics apparent [Brown 1987]. The pointer changed according to the link type if it hovered over a link (Fig. 6). Since mouse pointers are independent from screen and text layout, this may be an interesting option for Web clients, too. Standard software, like word processors and graphics programs, and also operating systems, commonly employ these differently shaped mouse pointers as it is possible to indicate many different actions in a non-obtrusive, yet immediately visible manner. 


\section{Multiple endpoints}

Links with multiple endpoints do not connect only two, but a set of related nodes. Thus different alternative destinations can be provided. When a user initiates the traversal of a link with multiple endpoints, he can be requested to choose between the available options. This solution was preferred by most former hypertext systems. Microcosm and DLS presented a list of generated link targets on an intermediary page as the result of a user query [Hall, Davis, Hutchings 1996; Carr et al. 1995]. Intermedia displayed a dialogue box with a list of link titles.

Likewise, the preferred idea for XLink seems to be a popup menu [Halsey, Anderson 2000; DeRose 2000]. Though lists of targets are probably the most straightforward approach, they may slow down Web navigation. A user has to make an additional selection from the pop-up list each time he follows a link.

Multiple links can also be used to automatically select the most decent destination by applying a filter. Already the father of hypertext Vannevar Bush suggested filters for links. If the user follows a Guided Tour, links of the displayed documents should be hidden [Bieber 97; Bush 45]. Intermedia could filter links by link attributes and Hyper-G by user rights. It would be even more desirable to filter by semantic criteria like a user's task or profile.

\section{Complex Link Anchors}

Many Web usability guidelines confine the setting and the length of link markers, e.g. Nielsen recommends that link markers should be about 5 words long [Nielsen 2000]. This restriction is a concession to the limited link visualization potentials of current Web browsers, where extended link spans result in hardly readable underlined text regions. Hypertext systems that displayed links only on demand avoided these readability problems.

The XML linking standard allows arbitrary complex link anchors. As explained before, it is even possible to create discontinuous anchors, i.e., anchors that consist of several distinct regions. To the user this may appear like multiple anchors that share the same destination, which can be irritating. In Web system evaluations, already links that are displayed in more than one line have been found confusing, as the beginning and end of the anchor were not indicated by the browsers used [Spool et al. 1999].

Consequently, the extent of a link marker should be visualized. This is possible in recent Web browsers: the link marker can be highlighted if the mouse hovers over the link. However, the browser configuration has to be changed or an appropriate CSS must be defined.

\section{Overlapping Link Markers}

Link markers may overlap, either because an author creates two anchors at two intersecting text sections which are related to different destinations ${ }^{2}$, or because other authors create anchors overlapping with the link spans of the original author.

Hardly any current Web user will be familiar with the idea of overlapping link markers as they cannot be found on the Web or any popular hypertext system. Currently, it is not possible to create such constructs in HTML, since there is no way to distinguish different opening and closing anchor tags. This technological problem can easily be solved even with embedded links as Hyper-G's markup language HTF demonstrated. It used link identifiers to associate opening and closing link tags [Maurer 1996].

Nonetheless it is much harder to find a usable solution for the visualization of overlapping link spans. Harmony, Hyper-G's browser, used overlapping colored background boxes to mark the beginning and end of up to six overlapping markers (Fig. 5). But even two overlapping links are hardly readable and this method will finally fail if a larger number of anchors intersects: the increasing number of boxes will shrink to pixel height before they finally disappear.

You are now accessing the Hyper-G server at the IICM
(Institute for Information Processing and Computer Supported
New Media) of Graz University of Technology, Austria. The
IICM information server is the home server of J.UCS (Journal
of Universal Computer Science). Figure 7: Link Overlap in Harmony.

The user must also be able to choose a desired link in the overlapping section. Third-Voice (Fig. 5) displays a pop-up window where the user can pick the link to follow. Harmony lets the user first select an overlapping link by singleleft clicks and then follow it by a double-left click [Andrews 1996, p. 54]. Both solutions are not optimal, as the first one needs always two and the second one may even need an uncertain number of clicks to follow a link. The current version_of Hyper-G does not support overlapping links any more.'

\section{A VISION FOR IMPROVED HYPERTEXT USER INTERFACES}

The Web, undoubtedly the most successful distributed hypertext system ever, has despite its simplicity already serious usability problems. It must be prevented that this becomes worse when extended linking features are introduced.

We would like to revive a discussion by presenting ideas of an user interface strategy for extended links. To accomplish this we consolidated experiences of earlier hypertext research with established and innovative GUI techniques to create a consistent vision. These thoughts are widely based

6 Example: the phrase "psycholinguistics department" might be a link to the department home page, while another link explains the meaning of "psycholinguistics".

${ }^{7}$ HyperWave Information Server Version 5.5 uses HTML as markup language. 


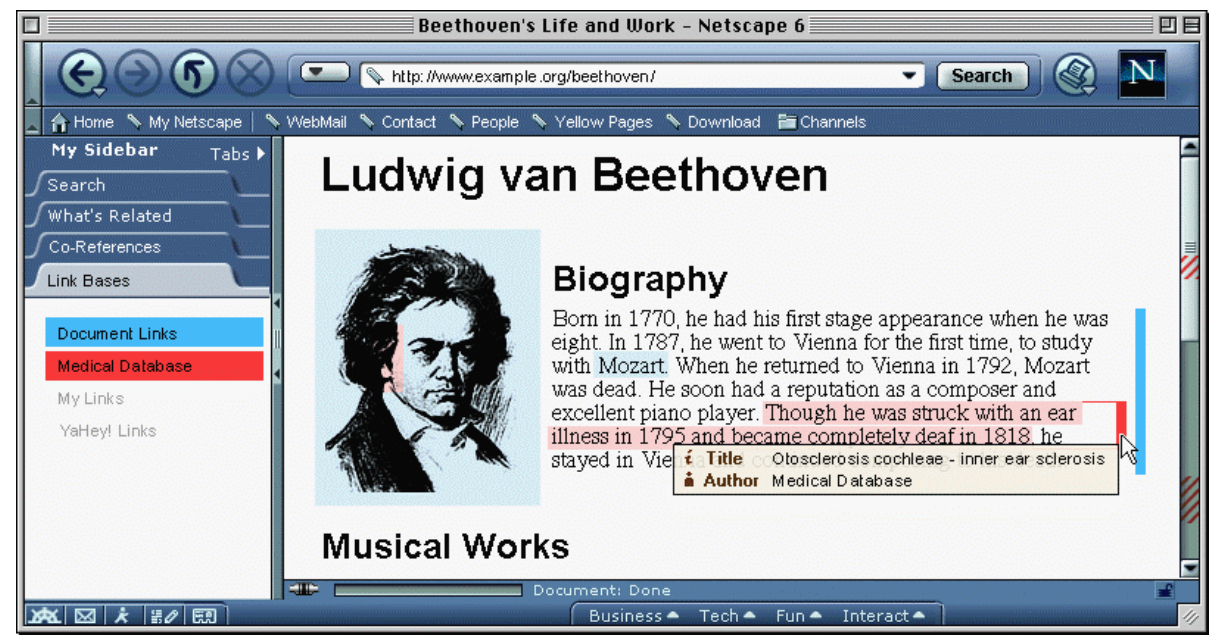

Figure 8: Mockup: Outgoing XLinks can overlap and are marked by transparency. Note the marked scrollbar.

on the analogy of the hypertext reader as a traveler, introduced in Landow's authoritative "Rhetoric of Hypertext" paper. He divides the interaction with links into two key parts: departure and arrival [Landow 1987].

Landow's ideas were based on his experiences with Intermedia. On the background of later hypertext research and the enriched linking capabilities of XLink a further discrimination is possible. The action of departure can be split into two sub-actions: first, the problem of locating the point of departure (identifying link markers) and second, the problem of getting sufficient information about the destination of the journey (understanding the link relationship). Considering the arrival procedure, the reader must get a reception at the destination to understand the extent and the context of the referenced material. The direction he came from, i.e., the origin of the journey, is the last page he visited and therefore known.

Finally, XLink does not only allow for links that connect just two endpoints - it is also possible to build XLinks that represent whole paths or structures. Thus, XLink at last embodies a standard Web storage format for structural information, e.g. for guided paths or for hierarchical site maps. We will discuss the uses of these hidden links (hidden in the sense that they are not originating from rendered page content) in a separate section.

\section{Point of Departure}

Current methods of Web authors - emphasizing text anchors by using color and style and using specially tailored graphics to mark graphical link anchors - are already so common that they will probably continue to exist when XLink is introduced. However, as illustrated above, these methods do not have the potential to identify extended or externally defined XLinks. Furthermore, no prevalent standard visualization method can be found to identify graphical or image map links. Consequently, new schemata are needed to display supplementary links, e.g. from an external linkbase provided by an XLink service.
From the usability point of view, a consistent and uniform technique is desirable, that does not distract from reading and does not interfere with text and graphical layout techniques, but enables the user to identify even complex anchors clearly. We think that an appropriate way to accomplish this might be the use of transparent areas overlaid on the hypertext document. Overlays have the advantage to be feasible with text and graphics, indicating active areas directly by masking them. They can be applied also at places where the document author did not plan a link. A possible distraction can be reduced by using soft and light colors for bright background and shady colors for dark background. User tests with more sophisticated transparent user interfaces showed promising results [Harrison 1995; Cox 1998].

An important factor that has to be considered is link density. If the ratio of marker area to unlinked area is high, the distinctive anchor appearance may overwhelm the "normal" text. Since an arbitrary number of "alien" links can relate to an XML Web page, a selection mechanism will have to prevent a phenomenon we would call "link overload", similar to information overload, which could overshadow the interaction potentials of the approaching XLink Web. Therefore, the user interface must provide means to select which links will be put on view.

Once more, this calls for links-on-demand display techniques. The selection mechanism may be provided by an additional tool bar or window. A "link database browser" could be displayed at the left side of the window like the history list of Microsoft Internet Explorer or the Sidebar of Netscape 6 . The tool would not only allow to select new link databases ${ }^{8}$, it would also permit to enable and disable

\footnotetext{
${ }^{8}$ XLink offers a standard storage mechanism for external links. This permits the construction of hyperbase systems that offer compiled collections of links, e.g. as the result of a query [Grønbaek \& Trigg 1999, p. 167]. These services could be provided just like today 's search engines or Web catalogues.
} 
linkbases, making them appear or disappear. Colors may be used to associate listed linkbases to the anchors on the screen (Fig. 8).

When a link starts from an anchor longer than a few words, the overall readability of the text rapidly decreases, at least as long as persistent highlighting is used. As with the introduction of XLink source anchors can become arbitrarily long, this question becomes increasingly important. We suggest a simple method to reduce the impact on readability: a narrow bar on the right side of the anchored paragraph. The use of different techniques for short and long anchors is suggested by looking at the use of conventional paper: markup on paper consists of highlighting words by coloring them with a transparent marker and, when longer passages need to be distinguished, marking whole paragraphs by using vertical lines on the page border. Sometimes a title is given to help recognize the underlying concepts of the passage. Markup of this style has the advantage to be apparent but not as distracting as long underlined text. It uses only little screen space and is goes along well with most layouts.

Since this simple technique does not show the exact location of link marker start and end, it should be supplemented by a rollover effect. The scrollbar, or optionally a smallscale overview window could be used to show the location of link anchors that are outside the currently visible page section. Using the scrollbar to locate particular areas on long Web pages was already suggested by [Laakso, Laakso $\&$ Saura 2000].

Overlapping links of several linkbases could be visualized by transparent overlays in a defined neutral color, like bright gray. If the user clicks on such an area, a transparent pop-up appears, showing a list of the available link titles in the color of their associated database. Moving the mouse over these transparent items will highlight the related link markers in the document.

\section{Destinations}

At first sight the more or less uniform looking links of the Web are not typed. Apart from the marked text, the only preview a user can always get in a Web browser is the destination URI [Spool et al 1999]. Even this scant information is frequently utilized by Web users. Sometimes link titles or alternative descriptions to graphics are provided to hint at the content of the target document.

Looking closer the current Web could already provide much richer information. Link targets can differ in type ("mailto:" links, downloads), availability (broken links), size, and connection speed (affecting download time). Further information of semantic nature like title, author and language of the target document or structural hints like indicating out-of-site links could be used to automatically enhance link preview. In a paper on our project HyperScout we already suggested techniques to display such information in pop-ups [Weinreich 2000].

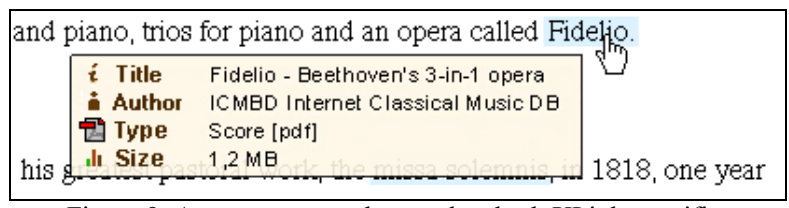

Figure 9: A pop-up menu that renders both XLink-specific, and other automatically gathered information.

While XLink's title information can also be straightforwardly displayed in such a pop-up window, the machine-readable information is provided to compute type information. This can be used to induce alternative traversal behavior, or to get advance information about file types of target documents. It could also be used to filter links according to a specified user profile. If this leads to alternative browser behavior, this must not be hidden from the user. In addition to pop-ups, we suggest the use of different mouse pointers to immediately indicate link actions, comparable to the method of the Guide system.

If an XLink offers several destinations, the problem of selection occurs. Pop-up menus with a list of available links are suggested in the XLink definition and some publications [DeRose 2000; Bosak and Bray1999]. They have, however, the disadvantage to require additional user action: the user has first to choose a link, click and then he has to choose a target anchor and click again. We would suggest to use the role attributes to allow filtering, thus displaying only part of the link targets available. In certain cases it might even be desirable that a default destrination is automatically selected when the left mouse button is clicked. Indicated by the mouse pointer, a pop-up appears only on right mouse click, presenting a choice of complementary link targets.

\section{Arrival}

The rhetoric of arrival in the sense of Landow requires that the reader gets the feeling of welcome at the destination document: "One must employ devices that enforce hypertext capacity to establish intellectual relations." [Landow 1987].

Establishing such an intellectual relation requires the user to determine the target of a link and its context. The method of today's Web browsers to present the target is simple: the whole document is shown, or, if a fragment identifier was specified, the browser tries to scroll to the position of the fragment anchor. In fact this is a known usability problem of the Web: as the span of the link target is not visualized the user cannot identify the extent of the destination. If the fragment locator is near the end of a page, the browser often cannot scroll sufficiently down to display the link target at the top of the window.

Tim Berners-Lee's first graphical Web browser on the NextStep system and later versions of Mosaic did already highlight the target anchor [Caillau \& Ashman 1999], a feature lost in current mainstream browsers. Since we already suggested transparent highlighting areas to identify starting anchors, a different method would be advisable to prevent misconceptions. 


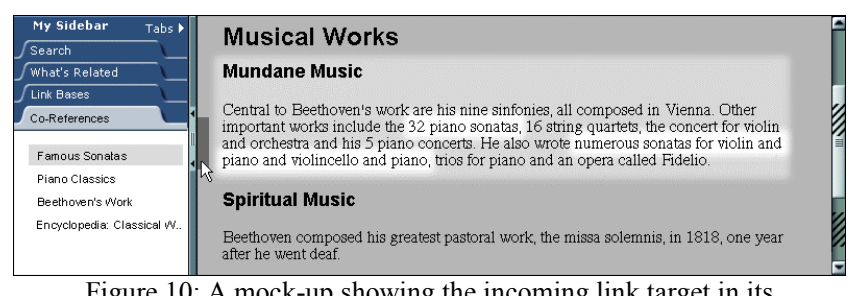

Figure 10: A mock-up showing the incoming link target in its context and information about co-references.

We again suggest a technique long before known to work on paper: lateral marker lines. A narrow bar on the far left side of the window is used to indicate the target span. The chance of confusing incoming and outgoing links is thus (incoming links: left vs. outgoing links: right) kept to a minimum. The Devise Hypertext System utilized a similar technique to indicate target anchors [Grønbæk \& Trigg 1999 , p. 314]. When a more precise visualization of the target becomes necessary (e.g. for tables), an on-demand method may be used: moving the mouse over the marker bar will shade the rest of the document except for the target area; a method already used in Harmony's PostScript viewer [Andrews 1996].

If the target section is larger than the visible window, clicking on the bar will "pin" the shading and the user may scroll the page. Additionally, the scrollbar may be used to show the extent of the target span compared to the whole page, especially useful if it does not fit into the window.

Although a very precise notion of the target anchor can be specified with XML linking, a weakness of XLink emerges: what the standard lacks, is a definition of the link context. Nanard and Nanard argue for a distinction of link anchor (as trigger) and link context (as minimum reading context) at both ends of the link. The link anchor is usually quite short and focused, as the link context is embedding the anchor, enabling the reader to understand the relationship of a link better [Nanard \& Nanard 1993; Hardman et al. 1994]. The meaning of a sequence of words can change severely when torn out of its context, i.e., the surrounding sentence, paragraph or chapter. XLink misses an explicit definition of such context spans. This is a serious disadvantage, that could be easily fixed by an additional attribute for the resource tags. Then, if an anchor is selected, the context should be made visible.

It is also sometimes useful to supplement other links pointing to the target anchor, called co-references. These links could have been collected in earlier sessions, retrieved from search engines or compiled by linkbase systems. They can stem from material that was not visited in the course of the current search, and, if followed in the reverse direction, they can provide material related to the current target anchor.

We suggest to make the most appropriate co-references of the last navigated link available by right clicking on the lateral marker bar, just as the right click opens a pop-up on link anchors. A double click can be used to open a larger list in the left side of the browser with more references pointing to that document. Finally, we think it would be feasible to apply filtering mechanisms utilizing the arc roles, e.g. to display only links that use the current anchor as an "example".

\section{The Use of Hidden Links}

So far we have tried to optimize the visualization of hyperlinks with markers in documents. However, XLink can also be used to describe relations without markers, e.g. nonassociative structural XLinks. These links can either be supplied by the author of a site or by an external source, e.g. a guided tour or a trail [Hammond \& Allinson 1987; Bush 1945]. Automatically generated link overview maps (local map, fisheye view, 3D landscapes) often seem to be more confusing than helpful, when used in large hyperspaces [Utting \& Yankelovich 1989]. Because of the immense size and the distribution of the Web, structural information has to be provided for an overview that truly can help to find semantically related content.

A special link type introduced by HTML 2.0 (<LINK REL $>$ ) to distinguish between structural and associative links [Berners-Lee 1995]. Though this made it possible to separate structure-related and content-related navigation, it is poorly supported by current Web browsers. Only some less widely spread browsers like Lynx and iCab (Fig. 10) support the use of structural links. Thus, so far there are only a handful of Web sites that offer structural links. Yet, this information could often be easily provided, especially for generated Web content or sites created with an authoring tool.

\section{围 $\square=0$} Figure 11: iCab's structural link navigation toolbar.

To support structural information in XLinks special link roles and arc roles would have to be defined. This, however, would use the role attributes not for semantic but rather for syntactical information. Then again, it would be possible to provide complete structures, e.g. Guided Tours or Site Maps, in a single link, something not possible with the LINK element.

As for link markers, a consistent interface is needed for structural navigation: we suggest that XLink-aware browsers should provide an iCab-like toolbar for basic structural navigation. Furthermore a hierarchical view, like HyperG's collection tree browser, can be provided on demand. This additional navigation tool should be displayed in the same browser window, e.g. in place of the sidebar. The interface should also provide a standard interface for Guided Tours or other meta-structures, thereby eliminating the need for workarounds.

We can also imagine hybrid XLinks which, bear structure, and have link markers in the Web page". This implicit structure could be extracted and displayed in the standard-

9 Such structural links include: links on a homepage pointing into the site, site logos pointing to the homepage, arrows for next and last pages, etc. 
ized user interface. The original embedded links should not be hidden: the user can thus either use the consistent standard interface (without having to search for navigation elements) or follow the rendered structural links (without having to leave the page context).

\section{CONCLUSION}

Usability has become a key factor for the success of software. Despite the intensive research on hypertext systems, no standard hyperlink user interface has been agreed on. We are thus bound to the de-facto standard of the Web, a design with many inherent weaknesses that does not agree with extended linking features.

Experiences from software engineering have shown how to do better: the initial design of a system has to include its user interface as well as its functionality [Nielsen 1993]. The representation of data is only of secondary importance.

This demonstrates the need of reconsidering currently developed standards: the XLink standard does hardly mention the user interface. The same lack of consideration of link interfaces is apparent in other W3C activities: Neither HTML nor its present descendants nor other standards like SMIL or the Semantic Web Initiative mention design issues regarding the user interface.

In this paper we try to stimulate a discussion on the visualization of and the interaction with extended hyperlink features. We believe that this is necessary to prevent an impairment of Web usability when new linking features are introduced.

Experiences from historical systems can help to avoid mistakes and to provide solutions that are still topical. This paper presents problems and solutions for the presentation of and the interaction with extended hyperlink features. Though we are aware that the developed vision can still be enhanced, we gathered well-tried methods to create a consistent and easy-to-use interface.

In this process, design issues for XLink arose: we found some open issues, i.e., the missing definition of contexts, default arcs, syntax attributes or attributes needed to carry preview information (like the size of a target document). Some issues were completely left out, like the distribution of links via linkbases or an exact specification for the use of the semantic attributes.

Nonetheless, XLink can be used even today: when XLinks are used on Web servers, the centralized storage makes link management much easier [Markos 2000]. Using XSL Transformations, XML or XHTML documents and XLink linkbases can be converted to HTML and be accessed by conventional browsers - right now.

In the long run, however, this functionality should be moved to the client - only then the browser will be able to exploit the full power of XLink. The success of XLink or a similar standard will eventually depend on two factors: decent tools for authors and readers.

\section{REFERENCES}

1. Andrews, K. (1996): Browsing, Building, and Beholding Cyberspace, New Approaches to the Navigation, Construction, and Visualisation of Hypermedia on the Internet, Ph.D. Thesis, Graz University of Technology, 1996, ftp://ftp.iicm.tu-graz.ac.at/pub/keith/phd/

2. Berners-Lee, T. (1989): Information Management: A Proposal. CERN Internal Communication, Graz, 1989, http://www.w3.org/History/1989/proposal.html

3. Berners-Lee, T. and Conolly, D. (1995): Hypertext Markup Language - 2.0, MIT / W3C, Boston / Graz, http://www.w3.org/MarkUp/html-spec/html-spec_toc.html

4. Bernstein, M. (1996): HypertextNow: Showing Links, Eastgate Systems Inc., 1996 http:/www.eastgate.com /HypertextNow/archives/ShowingLinks.html

5. Bieber, M., Vitali, F., Ashman, H., Balasubramaniam, V. and Oinas-Kukkonen, H. (1997): Forth Generation Hypermedia: Some Missing Links for the World Wide $W e b$. International Journal of Human Computer Studies, Vol. 47 (1), Academic Press, 1997, pp. 31-65

6. Bos, B., Lie, H. W., Lilley, C. and Jacobs, I. (Eds.) (1998): Cascading Style Sheets Level 2 Specification. World Wide Web Consortium, 1998, http://www.w3.org/TR/REC-CSS2/

7. Bosak, J. and Bray, T. (1999): XML and the SecondGeneration Web, Scientific American, May 1999, http://www.scientificamerican.com/1999/0599issue/05 99bosak.html

8. Brown, P. J. (1987): Turning Ideas into Products: The Guide System, Hypertext'87 Proc., ACM Baltimore, 1987, pp. 34-45

9. Bush, V. (1945): As We May Think, The Atlantic Monthly, July 1945, reprinted in: Interactions, Vol. III.2, 1996, pp. 35ff

10. Caillau, R. and Ashman, H. (1999): Hypertext in the Web - A History, ACM Computing Surveys, Vol. 31 (4), December 1999

11. Carr, L. , De Roure, D. , Hall, W. and Hill, G. (1995): The Distributed Link Service: A Tool For Publishers, Authors And Readers, Proc. 4th WWW Conference, Boston, Massachusetts, USA, 1995

12. Carr, L., De Roure, D., Hill, G. and Hall, W. (1996): Web Links as User Artefacts, Techreport, University of Southampton, UK, 1996

13. Chakrabarti, S., Gibson, D. A. and McCurley, K. S. (1999): Surfing the Web Backwards, In: Proc. of WWW 8 Conference, Toronto, Canada, 1999

14. Clark, J. (ed.) (1999): XSL Transformations (XSLT) Version 1.0, World Wide Web Consortium Recommendation, Nov. 1999, http://www.w3.org/TR/xslt

15. Clark, J. and DeRose, S. (eds.) (1999): XML Path Language (XPath) Version 1.0, WWW Consortium Rec., November 1999, http://www.w3.org/TR/xpath 
16. Conklin, J. (1987): Hypertext: An Introduction and Survey. IEEE Computer 20 (9), September 1987, pp. 17-40

17. Cox, D. A., Chugh, J., Gutwin, C. and Greenberg, S. (1998): The Usability of Transparent Overview Layers. Summary Proc. of CHI'98 Conference, ACM Press, 1998, pp. 301-302

18. Delisle, N., Schwartz, M.: Neptune: A Hypertext System for CAD Applications, Proc. of Conference on Management of Data, Washington, USA, 1986, pp. 132-143

19. DeRose, S., Maler, E. and Daniel, R. (eds.) (2001): XML Pointer Language (XPointer) Version 1.0, World Wide Web Consortium Reenter Last Call, January 2001, http://www.w3.org/TR/xptr

20. DeRose, S., Maler, E. and Orchard, D. (eds.) (2000): XML Linking Language (XLink), World Wide Web Consortium Proposed Recommendation, December 2000, http://www.w3.org/TR/xlink

21. DeRose, S.J. and Durand, D.G. (1994): Making Hypermedia Work: A User's Guide to HyTime, Kluwer Academic Publishers, Norwell, MA, USA, 1994

22. Grønbæk, K. and Trigg, R. H. (1999): From Web to Workplace, MIT Press, Massachusetts, USA, 1999

23. Halasz, F. G., and Schwartz, M. D. (1990): The Dexter Hypertext Reference, In: Proc. of Hypertext Standardization Workshop, Gaithersburg, USA, 1990, pp. 95-133

24. Hall, W., Davis, H. and Hutchings, G. (1996): Rethinking Hypermedia, The Microcosm Approach. Kluwer Academic, NL, 1996

25. Halsey, B. and Anderson, K. M. (2000): XLink and open hypermedia systems: a preliminary investigation, In: Proc. of ACM Hypertext Conference, San Antonio, USA, 2000, pp. $212-213$

26. Hammond, N. and Allinson, L. (1987): The Travel Metaphor as Design Principle and Training Aid for Navigating around Complex Systems. In: Proc. of the $3^{\text {rd }}$ Conference of the British Computer Society HCI Specialist Group, 1987, pp. 75-90

27. Hardman, L., Bulterman, D. and Rossum, G. (1994): Adding Time and Context to the Dexter Model, Communications of the ACM, Vol. 37 (2), Feb. 1994

28. Harrison, B. L., Kurtenbach, G. and Vicente, K. J. (1995): An Experimental Evaluation of Transparent User Interface Tools and Information Content, In: Proc. of User Interface Software and Technologies (UIST'95), Pittsburgh, USA, 1995

29. Laakso, S. A., Laakso, K.-P. and Saura, A. J. (2000): Improved Scroll Bars, CHI 2000 Extended Proceedings: Interactive Posters, Den Haag, 2000

30. Landow, G. P. (1987): Relationally Encoded Links and the Rhetoric of Hypertext. In: Hypertext'87 Proc., ACM, Baltimore, 1987, pp. 331-343

31. Lythgoe, J. N. (1979). The Ecology of Vision, Oxford University Press, Oxford, 1979
32. Markos, J. (2000): Out of Line Linking with X2X Satisfying the need for better access to information, White Paper, empolis UK limited, http://www.empolis.co.uk/technology/tech_lin.asp

33. Maurer, H. (1996): Hyper-G now Hyperwave: The next generation Web solution. Addison-Wesley, Harlow, UK, 1996

34. Nanard, J. and Nanard, M. (1993): Should Anchors be Typed Too? An Experiment with MacWeb. Proc. of ACM Hypertext '93 Conference,1993, pp 51-62

35. NCSA (1996): Mosaic(tm) for MS Windows User Guide, National Center for Supercomputing Applications (NCSA), University of Illinois, 1996, http://www.ncsa.uiuc.edu /SDG/Software/mosaic-w/releaseinfo/2.1/index.html

36. Nielsen, J. (1993): Usability Engineering, Academic Press San Diego, USA, 1993

37. Nielsen, J. (2000): Designing Web Usability: The Practice of Simplicity, New Riders Publishing, 2000

38. Shneiderman, B. and Kearsley, G. (1989): Hypertext HandsOn! An Introduction to a New Way of Organizing and Accessing Information. Addison-Wesley, Reading, MA, 1989

39. Spool, J., Scanlon, T., Schroeder, W., Snyder, C. and DeAngelo, T. (1999): Web Site Usability: A Designer's Guide. Morgan Kaufmann Publishers, 1999

40. Streitz, N., Haake, J., Hannemann, J., Lemke, A., Schuler, W., Schütt, H. and Thüring, M. (1992): SEPIA: A Cooperative Hypermedia Authoring Environment, In: Proc. of 4th ACM Hypertext Conference (ECHT'92), Milan, Italy, 1992, pp. 1122

41. Trigg, R. (1983): A Network-Based Approach to Text Handling for the Online Scientific Community, Ph.D. Thesis, Dept. of Computer Science, Univ. of Maryland, Nov. 1983. http://www.parc.xerox.com/spl/members/trigg /thesis/thesis-chap4.html

42. Utting, K. and Yankelovich, N. (1989): Context and Orientation in Hypermedia Networks, ACM Transactions on Office Information Systems (TOIS), Vol. 7, No. 1, January 1989

43. Vitali, F. and Bieber, M. (1999): Hypermedia on the Web: What Will It Take? ACM Computing Surveys 31(4), Dec. 1999, http://www.cs.brown.edu/memex/ ACM_HypertextTestbed/papers/57.html

44. Walker, J. H. (1987): Document Examiner: Delivery Interfaces for Hypertext Documents, Proc. of ACM Hypertext '87 Conference, Chapel Hill, USA, 1987, pp. 307-323

45. Weinreich, H. (2000): Concepts for improved visualization of Web link attributes, Computer Networks, Vol. 33, 2000, pp. 403-416

46. Yankelovich, N., Haan, J. B., Meyrowitz, N. K. and Drucker, S. M. (1988): Intermedia: The Concept and the Construction of a Seamless Information Environment, IEEE Computer, Vol. 21 (1), 1988, pp. 81-96 